\title{
rlme: An R Package for Rank-Based Estimation and Prediction in Random Effects Nested Models
}

by Yusuf K. Bilgic and Herbert Susmann

\begin{abstract}
There is a lack of robust statistical analyses for random effects linear models. In practice, statistical analyses, including estimation, prediction and inference, are not reliable when data are unbalanced, of small size, contain outliers, or not normally distributed. It is fortunate that rank-based regression analysis is a robust nonparametric alternative to likelihood and least squares analysis. We propose an $\mathrm{R}$ package that calculates rank-based statistical analyses for two- and three-level random effects nested designs. In this package, a new algorithm which recursively obtains robust predictions for both scale and random effects is used, along with three rank-based fitting methods.
\end{abstract}

\section{Introduction}

Rank-based procedures retain distribution-free estimation and testing properties. These procedures are much less sensitive to outliers than the traditional analyses when random errors are not normally distributed. Alternative robust score functions can be accommodated with the rank-based methods to protect analyses from influential observations in factor and response spaces. Also, the choice of these score functions could depend on the prior knowledge on error distributions. The Wilcoxon score function is fairly efficient for moderate to heavy-tailed error distributions. For example, rank-based procedures with Wilcoxon scores achieve up to $95 \%$ efficiency relative to least squares methods when the data are normal and are much more efficient than least squares methods for heavy tailed error distributions. These properties make the rank-based methods appealing. However, to our knowledge, statistical analyses for random effects models using the rank-based methodology have not yet been considered in any statistical package. This article proposes an R package with three rank-based fitting methods that estimate fixed effects and predict random effects in two- and three-level random effects nested models.

The rank-based norm, analogous to the least squares norm, is briefly defined as

$$
\|w\|_{\varphi}=\sum_{i}^{n} a\left[R\left(w_{i}\right)\right] w_{i}, w \in R^{n}
$$

where the scores are generated as $a(i)=\varphi[i /(n+1)]$ for a non-decreasing function $\varphi(u)$, defined on the interval $(0,1)$, and $R\left(w_{i}\right)$ is the rank of $w_{i}$ among $w_{1}, w_{2}, \ldots, w_{n}$. We assume without loss of generality that the scores sum to zero. Two of the most commonly used score functions are the Wilcoxon $\varphi(u)=\sqrt{12} \cdot\left(u-\frac{1}{2}\right)$ and the $\operatorname{sign} \varphi(u)=\operatorname{sgn}[u-1 / 2]$.

The rank-based estimate of $\beta$ for the independent error model $Y=X \beta+e$ is given by

$$
\hat{\beta}_{\varphi}=\operatorname{Argmin}\|Y-X \beta\|_{\varphi} .
$$

Assume that the errors are independent and identically distributed with a continuous density function $f(x)$. Under regularity conditions, $\beta$ is estimated by

$$
\begin{gathered}
\hat{\beta} \dot{\sim} N\left(\beta, \tau_{\varphi}^{2}\left(X^{T} X\right)^{-1}\right), \\
\tau_{\varphi}=\left[\int \varphi(u) \varphi_{f}(u) d u\right]^{-1},
\end{gathered}
$$

with $\varphi_{f}(u)=-\frac{f^{\prime}\left(F^{-1}(u)\right)}{f\left(F^{-1}(u)\right.}$. The parameter $\tau_{\varphi}$ is a scale parameter for the error terms $e$. The rank-based estimator of the fixed effects for independent linear models is asymptotically normal, shown in the work of Jaeckel and Jureckova in the 1970's. See Chapter 3, Hettmansperger and McKean (2011) for the relevant theory in detail. Recently, the Rfit package was released for rank-based regression analysis that uses rank-based norm and robust estimators for independent linear models (Kloke and McKean, 2012). We extend the rank-based regression methodology to include random effects nested models.

Random effects nested models are frequently utilized in many research areas such as: education, survey sampling, meta-analysis, agriculture, and health. Survey sampling might happen within organizational units, communities, clusters, or hospitals. The experimental design of interest is 
expressed in terms of fixed effects but, for these designs, nested factors are a natural part of the experiment. These nested effects are generally considered random and must be taken into account in the statistical analysis. For example, repeated measures design, randomized block design, and cluster correlated data consider each subject as a cluster having a correlated structure so the observations are nested within the subject. This subject would be a block, a cluster, an institution or a region. These designs are examples of two-level nested structures. Compound symmetric error structure is a natural variance-covariance form of these designs. It implies that all distinct members of a cluster or a subcluster are equally correlated with each other. When there is one more correlated level (subclusters) within clusters, this design could be called a three-level nested structure. Hierarchical linear models can also deal with these nested designs.

To illustrate the issues involved in data analysis, consider a simple example of a two-level nested design where students are nested within schools (see Model 5), and a three-level nested design where students are nested within sections in schools (see Model 6). Students are measured on a continuous univariate response variable of interest, $y$. The $p$-variate design data, including covariates, treatment, etc., are stored in the $x$ vector. The problem is summarized in the linear model as

$$
y_{i j}=\alpha+x_{i j}^{T} \beta+a_{i}+\epsilon_{i j}
$$

with $i=1, \ldots, I$ and $j=1, \ldots, n_{i}$, where $a_{i}$ is the random effect for school $i$ (cluster effects), $I$ is the number of schools, $n_{i}$ is the size of the $i^{\text {th }}$ school, and

$$
y_{i j k}=\alpha+x_{i j k}^{T} \beta+a_{i}+w_{j(i)}+\epsilon_{i j k}
$$

with $i=1, \ldots, I, j=1, \ldots, J_{i}$ and $k=1, \ldots, n_{i j}$, where $a_{i}$ is the random effect for school $i$ (cluster effects), $w_{j(i)}$ is the random effect for the $j^{\text {th }}$ section of school $i$ (subcluster effects), $J_{i}$ is the number of sections in school $i, n_{i j}$ is the size of $j^{t h}$ section in school $i$. Random errors are uncorrelated and independent.

The main interest with these models would be to estimate the regression parameters $\beta$ as fixed effects, to predict $a_{i}$ and $w_{j(i)}$ as random effects, and scale parameters of the error and random effects. The intra-class correlation coefficient (ICC) for each nested level would be also estimated using the scale parameter estimates. ICC provides information on the degree of dependencies of the observations within the same cluster. It is a useful and contextual parameter associated with the random effects of clusters that measures the proportion of the variability of the response to the total variability. It is sometimes called cluster effect and applied only to random models. For example, independent observations within- and between-cluster yield an ICC of zero.

This nested analog could be adopted for other organizational studies and hierarchical data. These designs often address questions related to

- the examination of differences within and across clusters or contexts such as classrooms, schools, neighborhoods, or groups on individual outcomes;

- the investigation of the degree to which individuals within a group or cluster are similar as measured through the ICC;

- the study of the factors that explain institutional/cluster differences;

- the effects of clusters and treatment on individual scores, e.g., student's academic achievement - both random and fixed effects are addressed in these interests.

In this article, three rank-based fitting methods and a new prediction algorithm are briefly introduced. A data analysis example using our package, rlme, is also presented.

\section{Three rank-based methods}

This section introduces three rank-based fitting methods to obtain fixed effects estimations: Joint Ranking (JR), Generalized Rank Estimate (GR) and Generalized Estimating Equation (GEER). The algorithm for robust variance estimates and random effects predictions in random effects nested models, called Rank Prediction Procedure (RPP), is then introduced. To sketch the calculation algorithms in these methods, Models (5) and (6) can be rewritten in the general mixed model matrix and vector notations as follows:

$$
Y=X \beta+e=X \beta+Z b+\epsilon,
$$

where $Y$ denotes a $n \times 1$ vector of responses, $X$ is a $n \times(p+1)$ known fixed effects design matrix, $\beta$ is a $(p+1) \times 1$ fixed effects parameter vector, $Z$ is a $n \times k$ known random effects design matrix, $b$ is a $k \times 1$ vector of random effects, and $\epsilon$ is a $n \times 1$ vector of random errors. 
Alternatively, the model can be written in vectors of the observations obtained from independent $I$ clusters. Within cluster $k$, let $Y_{k}, X_{k}$, and $e_{k}$ denote respectively the $n_{k} \times 1$ vector of responses, the $n_{k} \times p$ design matrix, and the $n_{k} \times 1$ vector of errors. Then the general mixed model for $Y_{k}$ is

$$
Y_{k}=\alpha 1_{n_{k}}+X_{k} \beta+e_{k}, k=1, \ldots I,
$$

where the components of the random error vector $e_{k}$ contain random effects and errors.

\section{Joint ranking method: JR}

This rank-based method for nested random effects models uses asymptotic results of the study by Kloke et al. (2009) in estimating fixed effects and standard errors. Kloke et al. (2009) developed the asymptotic theory for the rank-based estimates of fixed effects in the general mixed model using the general rank theory of Brunner and Denker (1994). The estimation of fixed effects in the JR method uses the dispersion function as in the independent linear model. However, the asymptotic distribution of $\hat{\beta}_{J R}$ has a different covariance matrix formula due to the correlated errors in the model. This is expressed as

$$
\operatorname{var}\left(\hat{\beta}_{J R}\right) \doteq \tau_{\varphi}^{2}\left(X^{\prime} X\right)^{-1}\left(\Sigma_{\varphi}\right)\left(X^{\prime} X\right)^{-1}
$$

where $\Sigma_{\varphi}=\lim _{I \rightarrow \infty} \sum_{i=1}^{I} X_{i}^{\prime} \Sigma_{\varphi, i} X_{i} \doteq \sum_{i=1}^{I} X_{i}^{\prime} \Sigma_{\varphi, i} X_{i}, \Sigma_{\varphi, i}$ is given by $\Sigma_{\varphi, i}=\operatorname{cov}\left(\varphi\left(F\left(e_{i}\right)\right)\right.$, and $F(x)$ denotes the distribution function of errors.

After estimating the fixed effects in Model (7), we predict the nested random effects and estimate the variance components using the random prediction procedure explained in the next section. In this method, for each cluster in Model (8), simple moment estimators of $\Sigma_{\varphi, i}$ is calculated as in Kloke et al. (2009).

\section{Iteratively reweighted generalized rank method: GR}

The generalized rank-based fitting for the general mixed model is an iteratively reweighted rank method based on the Newton-type approximation. Hettmansperger and McKean (2011) developed the asymptotic properties of linearized rank estimators for use in the linear model with the $k$-step Gauss-Newton approximation without weights. Bilgic (2012) and Bilgic et al. (2013) extended this theory to the $k$-step GR method in the general mixed models. After the first fitting, the estimates are asymptotically equivalent to the independent case because residuals are no longer dependent because of the reweighting with covariance weights. This algorithm could work for any type of variance-covariance error structure in the general mixed models.

Consider Model (7) where $\Sigma_{Y}$ is the variance-covariance matrix of the response vector $Y$ and $\theta$ is the vector of variance components of the model. The proposed iteratively reweighted generalized rank-based algorithm is as follows:

(0) Set $l=0$. The JR estimate serves as the initial fit.

(1) Obtain $\hat{\beta}^{(l)}$ as the rank-based fit of the model.

$$
Y^{*}=X^{*} \beta+e^{*},
$$

where $Y^{*}=\hat{\Sigma}_{Y}^{-1 / 2} Y, X^{*}=\hat{\Sigma}_{Y}^{-1 / 2} X$, and $e^{*}=\hat{\Sigma}_{Y}^{-1 / 2} e$. Thus, $\hat{\beta}^{(l)}$ minimizes the rank norm with $Y$ and $X$ replaced by $Y^{*}$ and $X^{*}$, respectively. If $l=0$ then use $\hat{\Sigma}_{Y}=I_{n}$; otherwise use $\hat{\Sigma}_{Y}=\Sigma_{Y}\left(\hat{\theta}^{(l-1)}\right)$.

(2) Use $\hat{\beta}^{(l)}$ to calculate the residuals, $\hat{e}^{(l)}=Y-X \hat{\beta}^{(l)}$.

(3) Use $\hat{e}^{(l)}$ to obtain $\hat{b}^{(l)}$, the predictor of $b$ via the RPP algorithm.

(4) Use $\hat{b}^{(l)}$ to estimate the variance components, $\hat{\theta}^{(l)}$ via the RPP algorithm.

(5) If $\left\|\hat{\beta}^{(l)}-\hat{\beta}^{(l-1)}\right\|<T O L_{1}\left\|\hat{\beta}^{(l-1)}\right\|$ and $\left\|\hat{\theta}^{(l)}-\hat{\theta}^{(l-1)}\right\|<T O L_{2}\left\|\hat{\theta}^{(l-1)}\right\|$ then stop. Else let $\hat{\beta}=\hat{\beta}^{(l)}, \hat{\theta}=\hat{\theta}^{(l)}$ and $\hat{b}=\hat{b}^{(l)}$. Set $l=l+1$ and return to step (1).

The estimators of the asymptotic variance-covariance matrix of $\hat{\beta}_{G R}$ require consistent $\tau_{\varphi}$ and $\Sigma_{Y}$ which are obtained from the current estimate of weighted errors.

\section{Rank-based generalized estimating equations method: GEER}

Considering an alternative representation of generalized linear models for correlated data in estimates, Abebe et al. (2013) extended the general estimating equations (GEE) method of Liang and Zeger 
(1986) for the general mixed models in the rank-based norm, and derived the asymptotic normality of the rank estimators. Briefly, as Abebe et al. (2013) describe, we can rewrite the general estimating equations expression proposed by Liang and Zeger in terms of the Euclidean norm in Model (11), and thus, the rank-based norm in Model (12) as follows:

$$
D_{G E E}(\beta)=\sum_{i=1}^{I}\left(Y_{i}^{*}-D_{i}(\beta)\right)^{2}
$$

and

$$
D_{G E E R}(\beta)=\sum_{i=1}^{I} \varphi\left[\frac{R\left(Y_{i}^{*}-D_{i}(\beta)\right)}{n+1}\right] \cdot\left[Y_{i}^{*}-D_{i}(\beta)\right],
$$

with $n_{i} \times 1$ vectors $Y_{i}^{*}=\hat{V}_{i}^{-1 / 2} \cdot Y_{i}$, the estimate of the covariance matrix of $Y_{i}$ is $\hat{V}_{i}$, and $D_{i}(\beta)=$ $\hat{V}_{i}^{-1 / 2} \cdot E\left[Y_{i}\right]$.

Abebe et al. (2013) developed a class of nonlinear robust estimators minimizing the rank-based norm of the residuals defined in the rank-based general estimating equations utilizing a 'working' covariance structure in the rank-based fitting as an analogue of the GEE. Thus, the estimate of $\beta_{G E E R}$ is obtained by the usual iterated reweighted least squares algorithm applied to the rank-based fitting in Equation (12).

\section{Theory references and comparison}

The asymptotic derivations for the proposed estimators are discussed in several papers; Kloke et al. (2009) for the JR method; Bilgic et al. (2013) for the GR method; and Abebe et al. (2013) for the GEER method. In these studies, the rank-based estimators are competitive with the traditional methods such as maximum likelihood (ML), restricted maximum likelihood (REML) and least squares in the normal case and outperform when random errors are contaminated and exhibit better efficiency properties of the estimates when outliers exist. Among the three methods, the JR method is unweighted so its empirical validity and efficiency for the fixed effects is reported to be poorer than the other two methods in the Monte Carlo study performed by Bilgic (2012). The empirical validity and efficiency of GR and GEER methods are reported to be very similar. The GR estimates and their standard errors are obtained from the rank-based norm properties, whereas the GEER combines the rank-based norm and least squares properties. For highly correlated data, the GR or GEER method would be preferred.

The rlme package uses the suite of $R$ functions ww developed by Terpstra and McKean (2005) that computes fixed estimates for the rank analysis based on Wilcoxon scores when needed for independent linear models and initial fits. We plan to use the subroutines of the Rfit package for the next version of our package because it allows the user to choose the estimation algorithm for general scores.

\section{Rank-based prediction procedure: RPP}

So far, fixed effects estimations are calculated with the JR, GR and GEER methods described in the previous section. This section introduces robust predictions for random effects and variance components for two- and three-level nested designs. Robust predictions of random effects have been discussed in several papers, including Groggel (1983), Groggel et al. (1988), Dubnicka (2004), and Kloke et al. (2009). These predictions based on clusters use robust scale estimators. However, these papers only handle two-level random nested designs.

To illustrate how our recursive algorithm works, let us consider a two-level nested structure. The random effects model is defined as

$$
y_{i j}=x_{i j}^{T} \beta+a_{i}+\epsilon_{i j}=x_{i j}^{T} \beta+e_{i j}
$$

for $i=1,2, \ldots, I$ and $j=1,2, \ldots, n_{i}$ (say, $I$ schools, $n_{i}$ students in each). $a_{i}$ and $\epsilon_{i j}$ are random cluster effects and error effects, respectively. We observe the values of $y_{i j}$ and $x_{i j}$, the variables $a_{i}$ and $\epsilon_{i j}$ are not observable. In cluster $i$, we rewrite (13) as $y_{i j}-x_{i j}^{T} \beta=e_{i j}=a_{i}+\epsilon_{i j}$. This is a location model. The residuals $\hat{e}_{i j}$ obtained from one of rank-based fittings predict the cluster effects $a_{i}$. Let $\hat{a}$ be a consistent location estimator. The next step is that the residuals $\hat{\epsilon}_{i j}$ are obtained from the subtraction $\hat{\epsilon}_{i j}=\hat{e}_{i j}-\hat{a}_{i}$. Hence, we are ready to estimate the scale parameters of the errors with a robust scale estimator. In the package, RPP has two options for location and scale estimates. The options are the median (med) and Hodges-Lehman $(H L)$ location estimators in Equation (14), and the median absolute deviation $(M A D)$ and the dispersion scale estimator (Disp) in Equations (15) and (16), respectively. In the prediction algorithm, one estimator from each is needed. Natural pairs are med-MAD and HL-Disp. 
For a vector $e$ of errors $e_{i}$ in $n \times 1$, the robust location estimate associated with the Wilcoxon scores is the $H L$ estimator expressed as

$$
H L(e)=\operatorname{med}_{s \leq t}\left\{\left(e_{s}+e_{t}\right) / 2\right\}
$$

Our scale estimators in the package are MAD and Disp defined as

$$
M A D(e)=1.483 \operatorname{med}_{i}\left|e_{i}-\operatorname{med}_{j}\left\{e_{j}\right\}\right|
$$

and

$$
\operatorname{Dis} p(e)=\frac{2 \sqrt{\pi}}{n} \sum_{i=1}^{n}\left(\frac{R\left(e_{i}\right)}{n+1}-\frac{1}{2}\right) \cdot e_{i}
$$

The estimator in Equation (16) is a consistent estimator of a scale parameter when the errors have a normal distribution (Hettmansperger and McKean, 2011). Kloke et al. (2009) suggest that the MAD is a robust and consistent estimator for scale parameter in clustered correlated designs.

For a three-level nested structure, consider Model (6). The residuals $\hat{e}_{i j k}$ obtained from the fit predict $a_{i}+w_{i j}$ using the location model

$$
y_{i j k}-x_{i j k}^{T} \beta=e_{i j k}=a_{i}+w_{i j}+\epsilon_{i j k} .
$$

To separate the cluster effects $a_{i}$ and the subcluster effects $w_{i j}$, we need location estimates for each subcluster $w_{i j}$, which is nested within the cluster $a_{i}$. Proceeding over all subclusters using one of our robust location estimators, the estimates of $w_{i j}$ are obtained. After subtracting these from the residuals $\hat{e}_{i j k}$, it yields the estimates of the cluster effects $a_{i}$ using the location model $e_{i j k}-w_{i j}=a_{i}+\epsilon_{i j k}$. The scale parameters of the errors of each type, i.e. $a_{i}, w_{i j}$ and $\epsilon_{i j k}$, are then estimated with our robust scale estimators.

The RPP algorithm can handle k-level nestings in a hierarchical structure in the same manner. The algorithm needs only residuals from the model fitting for predictions of the random effects. In $k$-level random effects nested models, these residuals contain the estimates of errors and random effects. Groggel (1983) calls these random effects pseudo-samples, which are formed using consistent location estimators in this recursive way. Pseudo-samples are asymptotically equivalent to the true random effects in the model. See Bilgic (2012) for details.

\section{Data example}

The rlme package uses linear model syntax in the lme 4 package for two- and three-level models. To illustrate how our package does nested structured data analysis, a data set was obtained from the OECD Programme for International Student Assessment (PISA) conducted in 2009 (OECD, 2010). The data set includes 334 observations of metacognitive scores in 11 private schools in four geographic regions in USA. Metacognitive score is an index measure of the metacognitive aspect of learning. The research questions to be answered are how metacognitive scores depend on gender and age, and how the variability of the scores are explained by regional differences and school differences. Student scores are nested in the private schools which are nested within the regions. Data are correlated within region and school, hence, regions and schools are random effects on observations. This design would be considered hierarchical with two- or three- levels, such as students nested in regions, or students in schools nested within regions. In the package, this data set is called schools.

In our package, a 3-level nested design data analysis is done using the following syntax:

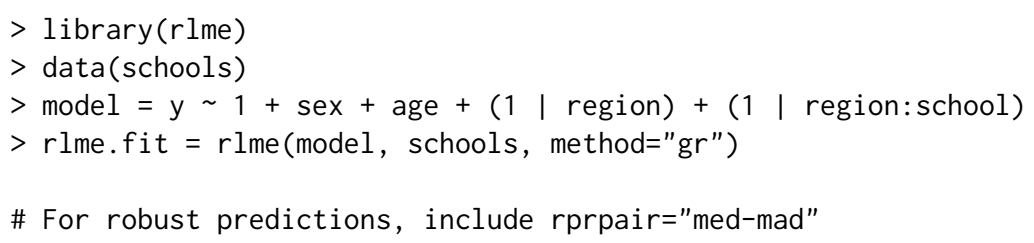

The formula syntax, the same as in the lmer function, expects the random effect terms to be enclosed with parenthesis, with nesting of variables denoted by a colon. In this example, region and school are two random effects, with school nested within region. The method is set to the rankbased method gr along with the prediction method hl-disp and Wilcoxon scores wil (the default). Alternatively the other rank-based fitting methods, jr or geer, and the maximum likelihood methods, reml or $\mathrm{ml}$, may be called from method.

The calculated fit can be examined using the summary function: 
Stand. Residuals vs. Fits in GR

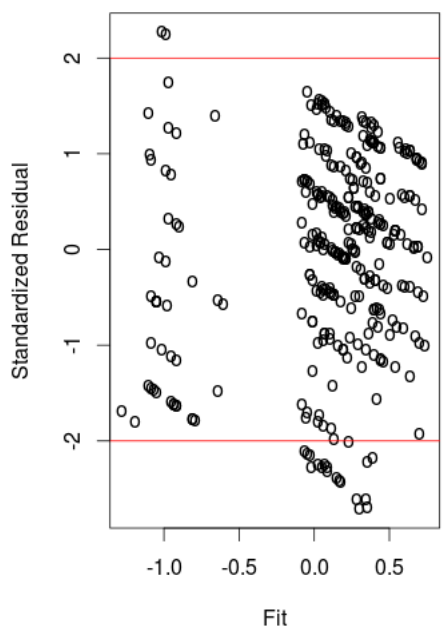

Normal Q-Q Plot

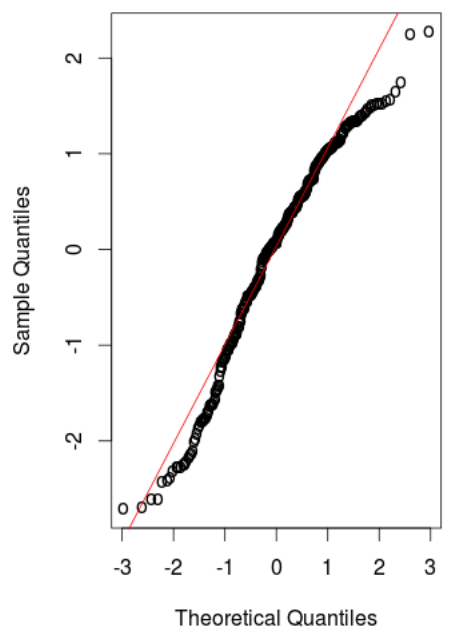

Figure 1: Standardized Residuals and QQ Plots.

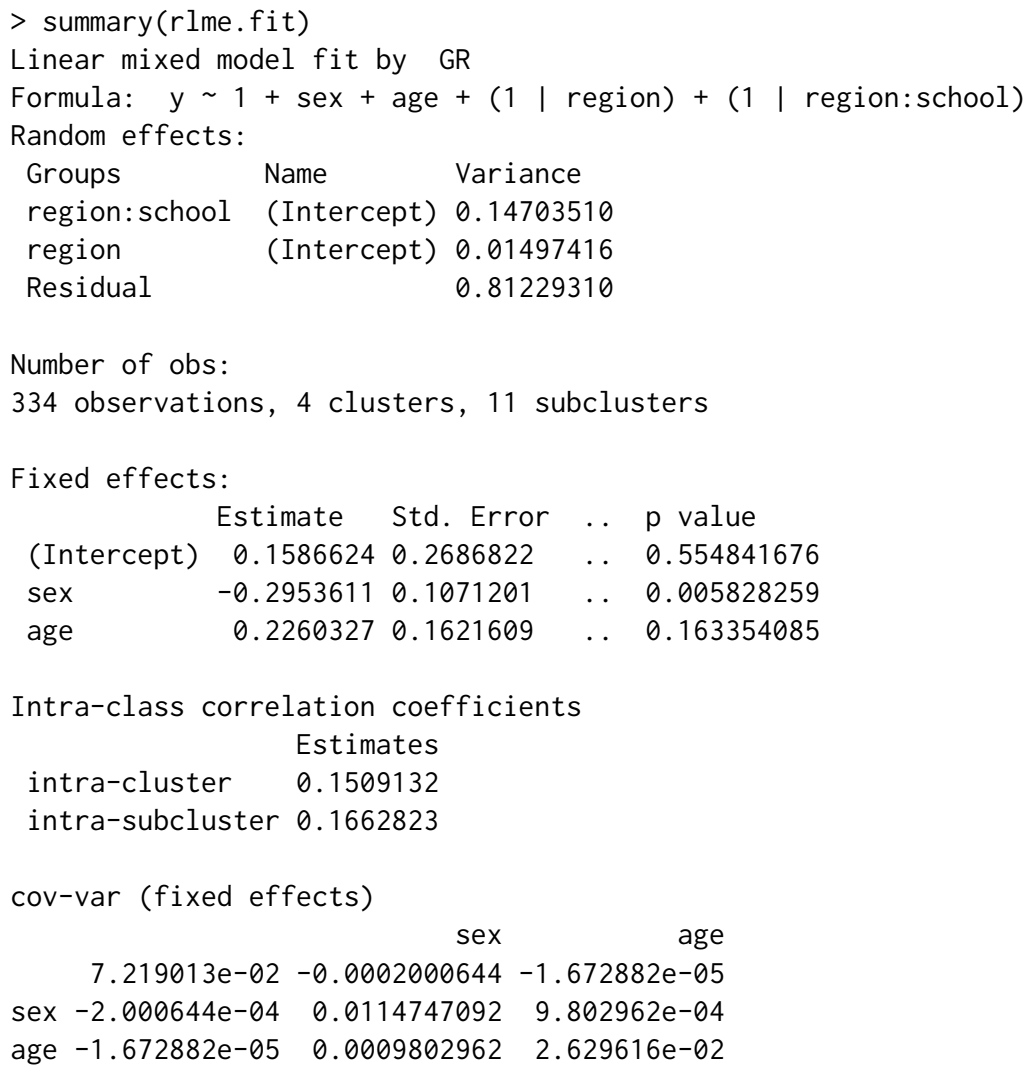

Here, intra-cluster, $\rho_{\text {region }}$, is the robust estimate of the intra-class correlation coefficient for region and intra-subcluster, $\rho_{\text {school(region) }}$, is for school nested within region. We can say that regional differences explain only $15.1 \%$ of the total variability in the model. The difference, $16.6 \%-15.1 \%=1.5 \%$, shows that the contribution of the school variability to the total variability could be ignored. Using the reml method, this result is calculated around $2.1 \%$, similar to the result of the rank analysis.

The plot function can be used to generate the standardized residuals vs. the fitted response and a normal Q-Q plot as shown in Figure 1:

$>\operatorname{plot}($ rlme.fit)

The residuals and random effects can be extracted from the fit. For example, we can extract the raw residuals through the list element ehat: 


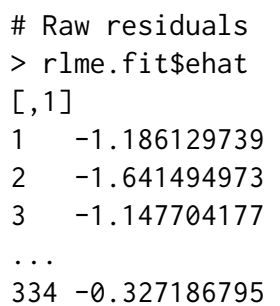

Several other elements of interest would include the effects estimates/predictions of the fixed effects, errors, clusters, and subclusters, which are obtained from rlme.fit $\$$ fixed.effects, . \$effect.err, .. \$effect.cluster, and . . \$effect. subcluster, respectively. A full list can be found in the help( $r$ lme) command or the str command. The same model can be also evaluated with the likelihood methods, reml or $\mathrm{ml}$ :

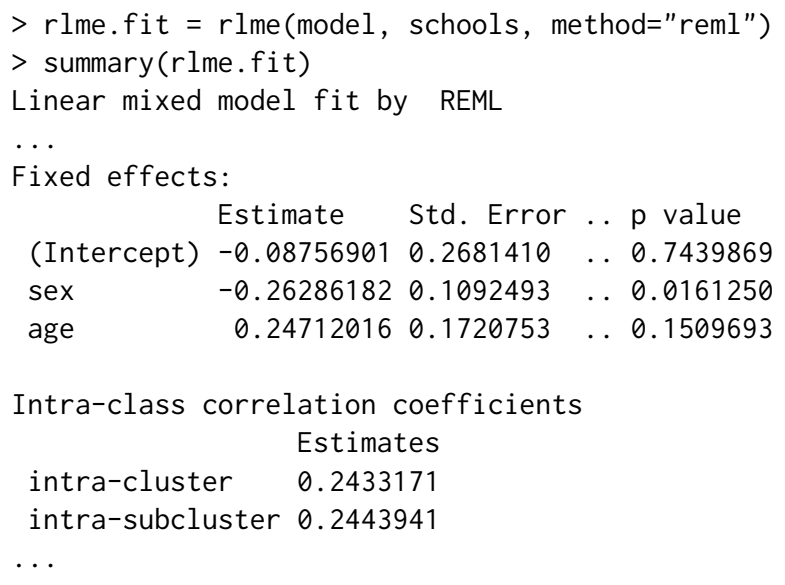

The REML and GR results are slightly different, but do coincide in the inference at the 5\% level. A 2-level random effects nested data analysis that students are nested within regions can be done in a similar syntax:

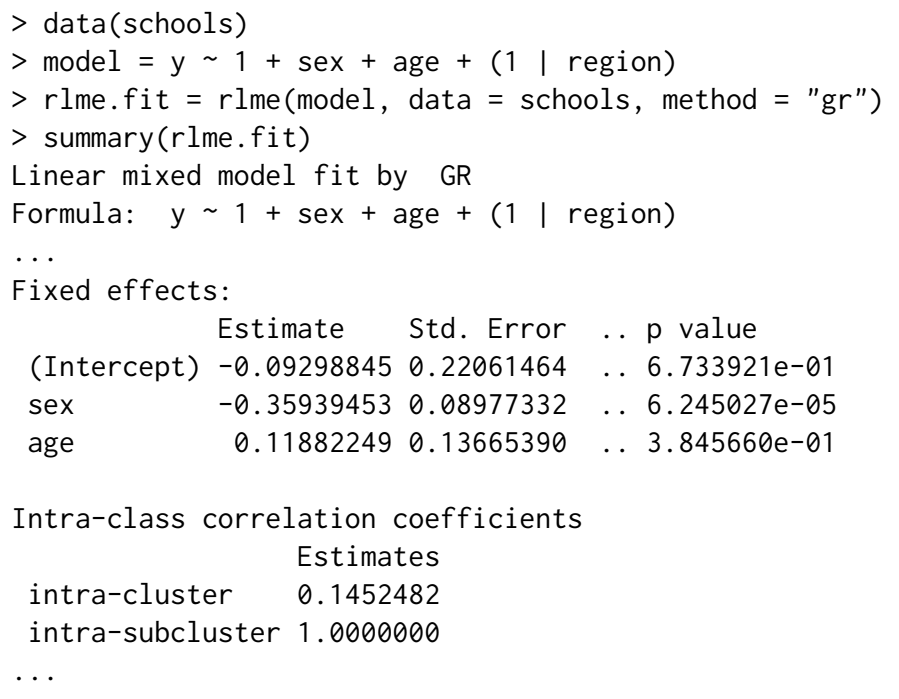

Diagnostics, TDBETAS and CFITS, to detect differences in fits for various methods can be obtained with the function fitdvcov. To compare the fixed effects estimates from any two fits, the covariance matrix from one rank-based method is required. Here we compare the REML and GR methods for the model:

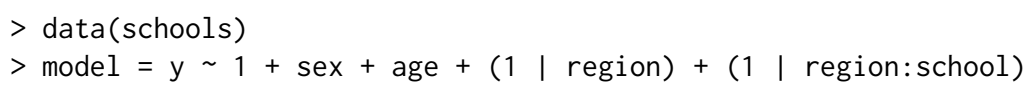




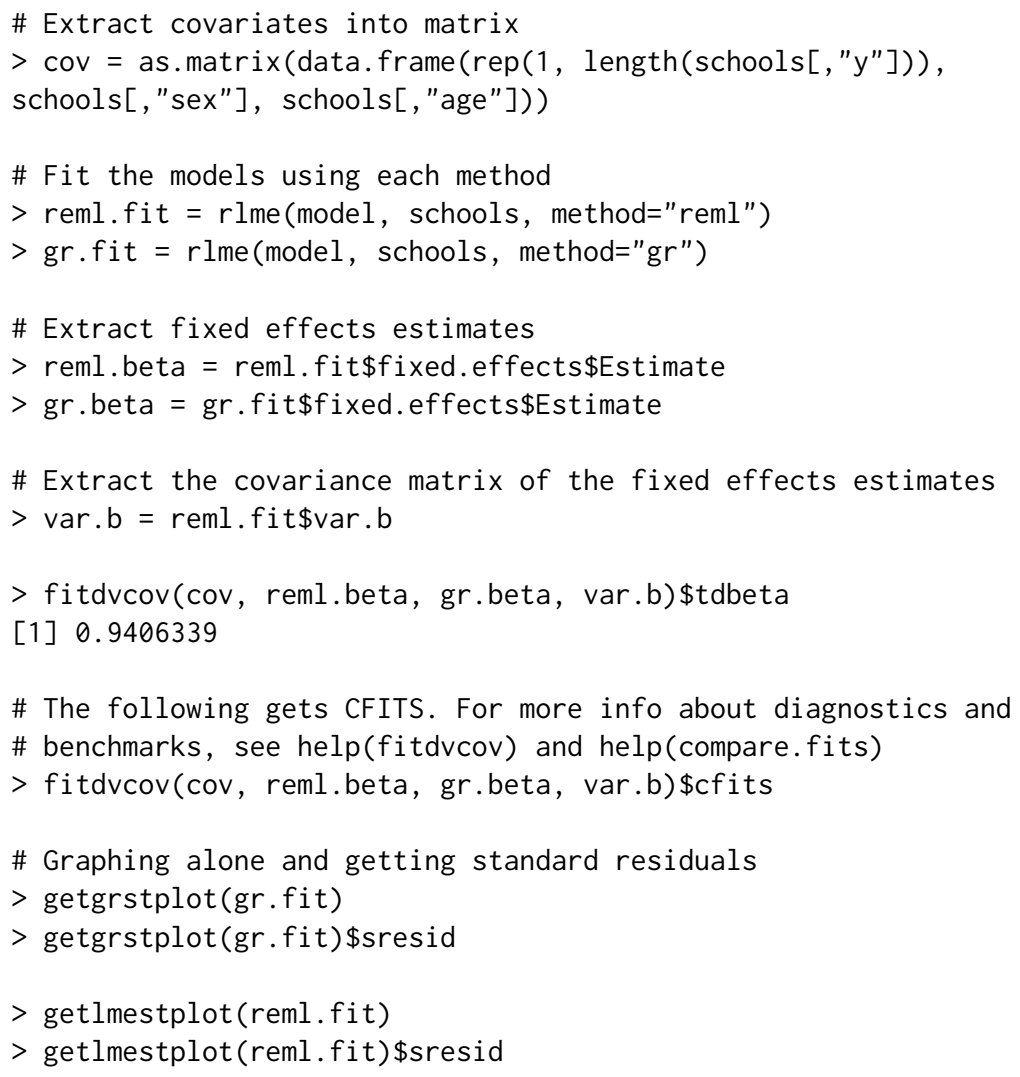

In our package, in case of potential outliers in factor space, high breakdown weights (hbr), weight="hbr", are specified along with method="geer". We use the routines of the $w w$ R codes for independent models:

$>\operatorname{data}($ schools $)$
$>\operatorname{rlme} . f i t=$ rlme $(y \sim 1+$ sex + age, data $=$ schools $)$

You have entered an independent linear model.

Continuing using the ww package.

Wald Test of $\mathrm{H} 0$ : BETA1 $=\mathrm{BETA} 2=0$

TS: 38.4414 PVAL: 0

Drop Test of $\mathrm{H} 0$ : $\mathrm{BETA} 1=\mathrm{BETA} 2=0$

TS: 7.819 PVAL: $5 \mathrm{e}-04$

\begin{tabular}{lrrrr} 
& EST & SE & \multicolumn{2}{c}{ TVAL PVAL } \\
BETA0 & 0.2333 & 0.0000 & 9105.8041 & 0 \\
BETA1 & -0.4192 & 0.0478 & -8.7664 & 0 \\
BETA2 & 0.0000 & 0.0772 & 0.0000 & 1
\end{tabular}

\section{Summary and further directions}

The rlme package analyzes random effects nested models with respect to estimation, inference and prediction for two- and three-level designs. These designs are a class of mixed models. Hierarchical linear model users may be interested in our package for robust analysis as well.

In the rlme package, estimations and inferences are obtained from three different rank-based methods along with the likelihood methods obtained from the nlme package (Pinheiro et al., 2013). These three methods include the joint ranking (JR), iteratively reweighted generalized rank (GR) and rank-based generalized estimating equations (GEER). Variance estimates and random effects predictions are obtained along with a robust algorithm, called Rank Prediction Procedure (RPP). New rank-based estimators are employed in these algorithm and methods. Diagnostics are included in our package as well. 
We are planning to extend statistical analysis of iteratively generalized rank-based methods using rank-norm properties to the general mixed models with various error structures. We would welcome any feedback and/or collaborations.

\title{
Acknowledgement
}

We would like to thank J. W. McKean for his invaluable guidance in theory and C. Leary for reviewing a preliminary version of this article.

\section{Bibliography}

A. Abebe, J. W. McKean, J. D. Kloke, and Y. K. Bilgic. Iterated reweighted rank-based estimates for GEE models. Under revision, 2013. [p73, 74]

Y. K. Bilgic. Rank-based estimation and prediction for mixed effects models in nested designs. PhD dissertation, Western Michigan University, Department of Statistics, 2012. [p73, 74, 75]

Y. K. Bilgic, J. W. McKean, J. D. Kloke, and A. Abebe. Iteratively reweighted generalized rank-based method in mixed models. Manuscript, 2013. [p73, 74]

E. Brunner and M. Denker. Rank statistics under dependent observations and applications to factorial designs. Statist. Plan. Infer, 42:353-378, 1994. [p73]

S. R. Dubnicka. A rank-based estimation procedure for linear models with clustered data. Journal of Modern Applied Statistical Methods, 3(1):39-48, 2004. URL http: //www. amstat. org/publications/ jcgs/. [p74]

D. J. Groggel. Asymptotic nonparametric confidence intervals for the ratio of scale parameters in balanced one-way random effects models. PhD dissertation, University of Florida, Department of Statistics, 1983. $[\mathrm{p} 74,75]$

D. J. Groggel, D. Wackerly, and P. Rao. Nonparametric estimation in one-way random effects models. Communications in Statistics: Simulation and Computation, 17:887-903, 1988. [p74]

T. P. Hettmansperger and J. W. McKean. Robust Nonparametric Statistical Methods. Chapman \& Hall, 2011. $[\mathrm{p} 71,73,75]$

J. Kloke and J. McKean. Rfit: Rank Estimation for Linear Models, 2012. URL http: //CRAN. R-project. org/package=Rfit. R package version 0.14 . [p71]

J. Kloke, J. W. McKean, and M. Rashid. Rank-based estimation and associated inferences for linear models with cluster correlated errors. Journal of the American Statistical Association, 104(485):384-390, 2009. $[\mathrm{p} 73,74,75]$

K. Y. Liang and S. L. Zeger. Longitudinal data analysis using generalized linear models. Biometrika, 73: 13-22, 1986. [p73]

J. Pinheiro, D. Bates, S. DebRoy, D. Sarkar, and R Core Team. nlme: Linear and Nonlinear Mixed Effects Models, 2013. R package version 3.1-109. [p78]

J. T. Terpstra and J. W. McKean. Rank-based analysis of linear models using R. Journal of Statistical Software, 14(7):1-26, 7 2005. ISSN 1548-7660. URL http://www. jstatsoft. org/v14/i07. [p74]

\author{
Yusuf K. Bilgic \\ SUNY Geneseo \\ 1 College Circle, Geneseo, NY 14454, (585) 245-5384 \\ USA \\ yusuf.k.bilgic@gmail.com \\ Herbert Susmann \\ SUNY Geneseo \\ 1 College Circle, Geneseo, NY 14454, (585) 245-5384 \\ USA \\ herbps10egmail.com
}

